\title{
An Analytic Expression of Thermal Diffusion Coefficient for the Hydrodynamic Simulation of Semiconductor Devices
}

\author{
TING-WEI TANG ${ }^{\mathrm{a}, *}$, XINLIN WANG $^{\mathrm{a}}$, HAITAO GAN ${ }^{\mathrm{a}, \dagger}$ and MEIKEI IEONG ${ }^{\mathrm{b}}$ \\ ${ }^{\mathrm{a}}$ Department of Electrical and Computer Engineering, University of Massachusetts, Amherst, MA 01003, USA; \\ ${ }^{\mathrm{b}}$ IBM SRDC East Fishkill Facility, Hopewell Junction, NY 12533, USA
}

\begin{abstract}
A new analytical expression of thermal diffusion coefficient $D^{T}$ is derived. To the firstorder approximation, it is given by $(1+\eta)^{-1}\left(D / T_{n}\right)$ rather than $(1-\eta)\left(D / T_{n}\right)$ where $\eta=-\left(T_{n} / \mu^{*}\right)\left(\partial \mu^{*} / \partial T_{n}\right)$ and $\mu^{*}$ represents the temperature-dependent bulk mobility. This new transport coefficient is implemented in our 2-D hydrodynamic device simulator and it seems to produce more reasonable results.
\end{abstract}

Keywords: Hydrodynamic simulation; Thermal diffusion coefficient; Effective carrier energy; Nonlocal mobility model

\section{INTRODUCTION}

Since the original derivation of hot-carrier transport model by Stratton [1], there has been controversy about what the proper transport coefficient of the thermal diffusion current density is [2-5]. In the hydrodynamic (HD) transport model, in addition to the diffusion current due to the carrier density gradient, there is also a thermal diffusion current driven by the carrier temperature (or energy) gradient. In most semiconductor devices, the thermal diffusion current is often in opposite direction to the carrier diffusion current. Therefore, improper use of the thermal diffusion coefficient in the HD transport model often affects the outcome of device simulation result. The purpose of this paper is to provide an analytical expression for the thermal diffusion coefficient which is more consistent with Monte Carlo (MC) simulation data.

\section{TWO FORMULATIONS OF TRANSPORT MODEL}

In general, there are two different formulations of the HD transport model [6]. Stratton's original formulation of the current density is given by [1]

$$
\vec{j}_{n}=q n \mu^{*} \vec{E}+q \nabla(n D) .
$$

\footnotetext{
* Corresponding author. Tel.: (413) 545-0763, Fax: (413) 545-4611, e-mail: ttang@ecs.umass.edu

${ }^{\dagger}$ Present address: Stanford University, Stanford, CA 94305.
} 
Assuming that the Einstein relation $D=\mu^{*} k_{B} T_{n} / q$ is valid and the bulk (homogeneous) mobility $\mu^{*}\left(T_{n}\right)$ is a known function of the electron temperature $T_{n}$, Eq. (1) becomes

$$
\begin{aligned}
\vec{j}_{n}= & q n \mu^{*} \vec{E}+\mu^{*} k_{B} T_{n} \nabla n \\
& +n \mu^{*}\left(1+\frac{T_{n}}{\mu^{*}} \frac{\partial \mu^{*}}{\partial T_{n}}\right) k_{B} \nabla T_{n} .
\end{aligned}
$$

We may cast the diffusion current density into the form

$$
\vec{j}_{\text {diff }}=q D \nabla n+q n D^{T} \nabla T_{n}
$$

where $D^{T}$ represent the thermal diffusion coefficient. Then, Stratton's formulation gives

$$
D^{T}=\frac{D}{T_{n}}\left(1+\frac{T_{n}}{\mu^{*}} \frac{\partial \mu^{*}}{\partial T_{n}}\right) .
$$

In our earlier work which is based on moments of the Boltzmann transport equation [7], we proposed a current density of the form [8],

$$
\vec{j}_{n}=\mu^{*}\left[q n \vec{E}+U \nabla n+\left(1-\lambda_{p}\right) n \nabla U\right]
$$

where $U=\langle\vec{\nu} \hbar \vec{k}\rangle \cdot \stackrel{\leftrightarrow}{1}$ and $\lambda_{p}$ is a dimensionless parameter extractable from the MC simulation. The origin of $\lambda_{p}$ comes from the modeling of the momentum collision moment $\vec{C}_{p}=\left\langle\hbar \vec{k}(\delta f / \delta t)_{\text {coll }}\right\rangle$ as

$$
\vec{C}_{p}=-\frac{q \vec{V}}{\mu^{*}}+\lambda_{p} \nabla U
$$

It should be noted that Eq. (5) becomes Eq. (2) if $\lambda_{p}=-\left(T_{n} / \mu^{*}\right)\left(\partial \mu^{*} / \partial T_{n}\right)$. However, the MC simulation data does not quite agree with this relation [9]. In the following, we shall present an analytical expression of $\lambda_{p}$ which is more consistent with the MC data.

\section{DERIVATION OF TRANSPORT PARAMETER $\lambda_{P}$}

Based on a series of MC simulations of $\mathrm{n}^{+}-\mathrm{n}-\mathrm{n}^{+}$structures [8], we have discovered that to capture the non-local effect, the carrier mobility is best described by an effective carrier energy (or temperature) defined by $[10,11]$

$$
W_{\text {eff }}=-\frac{q}{\delta_{\varepsilon}} \frac{\vec{j}_{\varepsilon} \cdot \vec{j}_{n}}{\left|\vec{j}_{n}\right|^{2}}
$$

where the simplified energy flux density $\vec{j}_{\varepsilon}$ is given by $[11,12]$

$$
\vec{j}_{\varepsilon}=-\delta_{\varepsilon}\left(\frac{\vec{j}_{n}}{q}\right) W-\Delta_{\varepsilon} \frac{\mu^{*} n W}{q} \nabla W
$$

and $W \approx(3 / 2) k_{B} T_{n}$ represents the average electron energy (see Fig. 1). The thermal conductivity coefficient $\Delta_{\varepsilon}$ and Peltier coefficient $\delta_{\varepsilon}$ are related to each other through [12]

$$
\Delta_{\varepsilon}=\delta_{\varepsilon}\left[\left(1-\lambda_{\varepsilon p}\right) \frac{1}{(W+U)} \frac{\partial R}{\partial W}-\left(1-\lambda_{p}\right) \frac{\partial U}{\partial W}\right]
$$

where $R=\langle\varepsilon \vec{\nu} \hbar \vec{k}\rangle \cdot \stackrel{\leftrightarrow}{1}$, and $\lambda_{\varepsilon p}$, is another dimensionless parameter extractable from the MC simulation. In the following, we assume first that $\lambda_{p} \approx \lambda_{\varepsilon p}$ which is supported by the MC data [8], and second, for simplicity, $R=(10 / 9) W^{2} \approx$ $(5 / 2)\left(k_{B} T_{n}\right)^{2}$ and $U=(2 / 3) W \approx k_{B} T_{n}$. These two approximations lead to a simplified relation

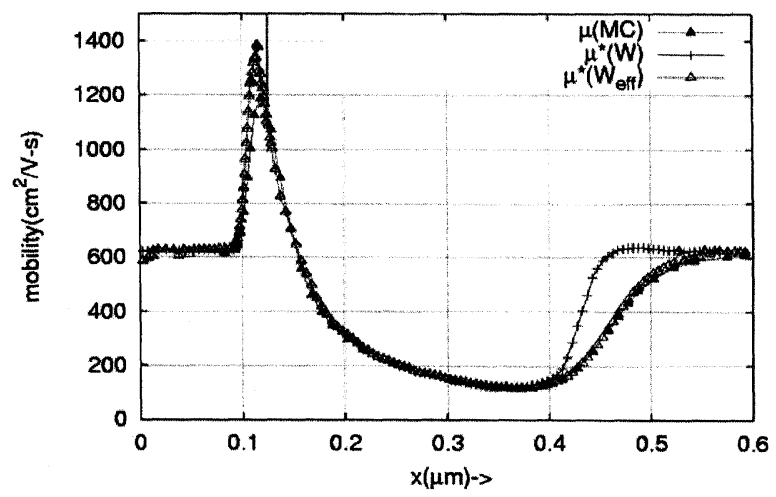

FIGURE 1 Comparison of the MC extracted mobility $\mu(\mathrm{MC}), \mu(\mathrm{W})$, and $\mu\left(\mathrm{W}_{\text {eff }}\right)$ for device $\mathrm{B}$ in Ref. [8]. $\mu\left(\mathrm{W}_{\mathrm{eff}}\right)$ fits better with $\mu(\mathrm{MC})$. 
between $\Delta_{\varepsilon}$ and $\delta_{\varepsilon}$ :

$$
\Delta_{\varepsilon} \approx(2 / 3) \delta_{\varepsilon}\left(1-\lambda_{p}\right)
$$

Equation (10) implies that the thermal diffusion coefficient $\Delta_{\varepsilon}$ of $\vec{j}_{\varepsilon}$ is related to the thermal diffusion coefficient $D^{T}$ of $\vec{j}_{n}$ through the factor $\left(1-\lambda_{p}\right)$.

In order to obtain an analytical expression of $\lambda_{p}$, we expand the reciprocal of the (inhomogeneous) mobility around the bulk (homogeneous) mobility and treat the difference between $\mathrm{W}_{\text {eff }}$ and $\mathrm{W}$ as a small perturbation. From Eq. (6) we have

$$
\frac{1}{\mu}=-\frac{\vec{V} \cdot \vec{C}_{p}}{q V^{2}}=\frac{1}{\mu^{*}}-\lambda_{p} \frac{\vec{V} \cdot \nabla U}{q V^{2}} .
$$

Substitution of Eq. (8) into Eq. (7) gives

$$
W_{\mathrm{eff}}-W=-\frac{\Delta_{\varepsilon}}{\delta_{\varepsilon}} \frac{W \mu^{*}}{q V^{2}} \vec{V} \cdot \nabla W .
$$

Expanding $1 / \mu$ around $1 / \mu^{*}$ and treating $\left(\mathrm{W}_{\mathrm{eff}}-\mathrm{W}\right)$ as a small perturbation, we obtain

$\frac{1}{\mu}=\frac{1}{\mu^{*}}+\frac{\partial}{\partial W}\left(\frac{1}{\mu^{*}}\right) \cdot\left(-\frac{\Delta_{\varepsilon}}{\delta_{\varepsilon}} \frac{W \mu^{*}}{q V^{2}} \vec{V} \cdot \nabla W\right)$

Comparing Eq. (11) and Eq. (13) and substituting Eq. (10) into Eq. (13) with the approximation $U \approx(2 / 3) W$, we finally arrive at

$$
\lambda_{p}=W \mu^{*} \frac{\partial}{\partial W}\left(\frac{1}{\mu^{*}}\right)\left(1-\lambda_{p}\right)=\eta\left(1-\lambda_{p}\right)
$$

where

$$
\eta=-\frac{W}{\mu^{*}} \frac{\partial \mu^{*}}{\partial W} \approx-\frac{T_{n}}{\mu^{*}} \frac{\partial \mu^{*}}{\partial T_{n}} .
$$

Hence,

$$
\lambda_{p}=\eta /(1+\eta)
$$

The physical meaning of Eq. (13) is clear. The energy-dependent mobility $\mu^{*}$ is strictly for bulk semiconductors only. However, if $W$ is replaced by

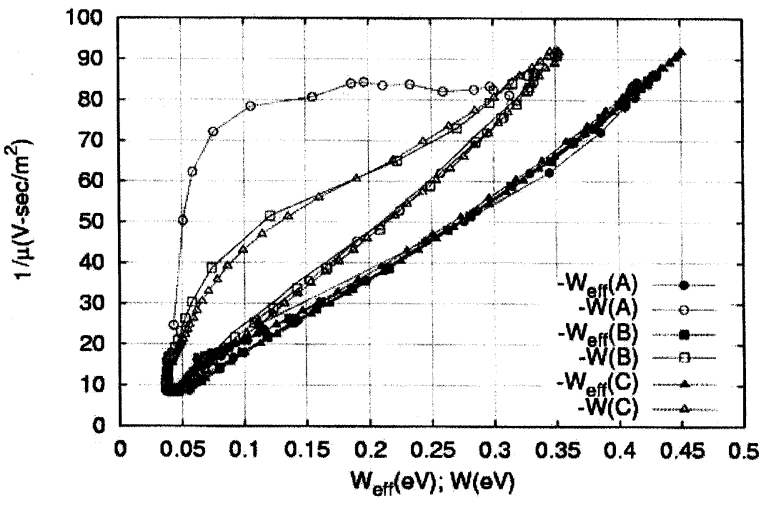

FIGURE $21 / \mu$ vs. $\mathrm{W}$ and $\mathrm{W}_{\text {eff }}$ for devices $\mathrm{A}, \mathrm{B}$, and $\mathrm{C}$.

$W_{\text {eff }}$ in $\mu^{*}$, this energy-dependent bulk mobility can also be extended to the case of inhomogeneous field, with a negligible "hysteresis" loop [11] (see Fig. 2). The second term on the r.h.s. of Eq. (13) represents a non-local correction to the bulk mobility.

An interesting feature of the result (14) is that the value of $\lambda_{p}$ never exceeds unity and therefore the positive definiteness of $D^{T}$ in Eq. (3) is guaranteed. This is not the case for $\lambda_{p}=\eta$ which is Stratton's model. Depending on what kind of the bulk mobility model $\mu^{*}\left(N_{i}, T_{n}\right)$ is used, if we assume $\lambda_{p}=\eta$, the value of $\lambda_{p}$ sometimes may exceed unity resulting in a negative $D^{T}$. Besides being unphysical, a negative $D^{T}$ often causes numerical instability.

\section{SIMULATING RESULTS}

We have implemented this new analytical expression of $\lambda_{p}$ in our 2-D HD simulator. A $0.1 \mu \mathrm{m}$ partially-depleted SOI-MOSFET given in Figure 2 of Ref. [12] is simulated. Figure 3 indicates a substantial spread in the $I_{D}-V_{D}$ characteristic curve among different models. In general, the model $\lambda_{p}=1\left(D^{T}=0\right)$ and the model $\lambda_{p}=\eta$ overestimate the drain current density because of $D^{T} \approx 0$. This result is due to the suppression of the thermal back diffusion in the hot-carrier region. On the other hand, the model $\lambda_{p}=\eta /(1+\eta)$ predicts slightly 


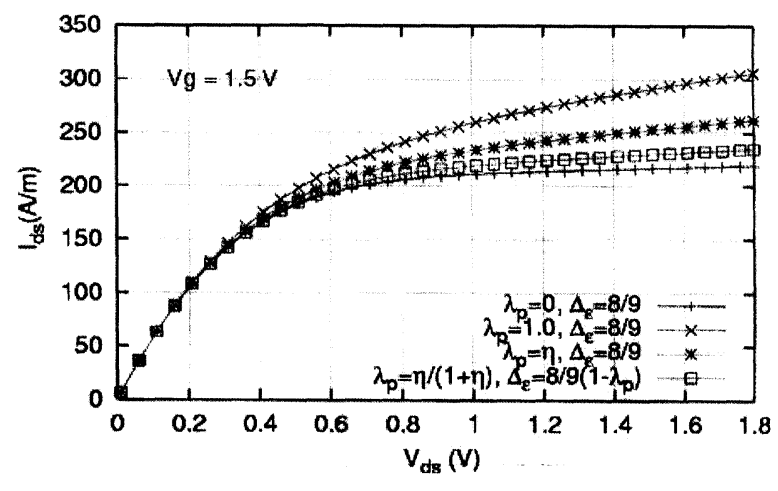

FIGURE $3 I_{\mathrm{ds}}-\mathrm{V}_{\mathrm{ds}}$ characteristics of the partially-depleted SOI-MOSFET at $\mathrm{Vg}=1.5 \mathrm{~V}$ for different $\lambda_{\mathrm{p}}$ 's.

larger current density than the conventional HD model $\left(\lambda_{p}=0\right)$. It is interesting to note that in the hot-carrier regime, $\eta$ has typically a value $\approx 1$ and thus our new analytical model predicts $D^{T} \approx(1 / 2)\left(D / T_{n}\right)$ rather than $D^{T} \approx 0$.

\section{CONCLUSION}

We have derived a new analytical expression for the coefficient of the thermal diffusion current density $D^{T}$. This derivation is based on an observation that a local energy-dependant bulk mobility can capture the non-local effect if the average electron energy is replaced by an effective average electron energy which is proportional to the ratio between the energy flux density and the charge flux density. To the first-order approximation, this leads to a simple expression $D^{T}=$ $(1+\eta)^{-1}\left(D / T_{n}\right)$ rather than $D^{T}=(1-\eta)\left(D / T_{n}\right)$, the result of Stratton's formulation. We have implemented this new analytical model in the 2-D simulation of a partially depleted SOI MOSFET. The simulation results indicate that $\mathrm{I}-\mathrm{V}$ characteristic curves are sensitive to the choice of the transport coefficient $\mathrm{D}^{\mathrm{T}}$.

\section{Acknowledgements}

This work was supported in part by the National Science Foundation under NSF Grant ECS9710463. The first author (T.-W. Tang) also acknowledges a support from the National Science Council through the National Center of HighPerformance Computing in Hsinchu, Taiwan, R.O.C.

\section{References}

[1] Stratton, R. (1962). "Diffusion of hot and cold electrons in semiconductor barriers", Phys. Rev., 126, 257-267.

[2] van Vilet, K. M. and Marshak, A. H. (1976). "Conduction current and generalized Einstein relations for degenerate semiconductors and metals", Phys. Stat. Sol. (b), 78, $501-517$.

[3] Lansberg, P. T. and Hope, S. A. (1977). "Two formulations of semiconductor transport equations", Solid-St. Electron, 20, $421-429$.

[4] Lansberg, P. T. (1984). "Dgrad( $\nu)$ or $\operatorname{grad}(\mathrm{D} \nu)$ ", J. Appl. Phys., 56, 1119-1122.

[5] Higman, J. and Hess, K. (1986). "Comment on the use of the electron temperature concept for nonlinear transport problems in semiconductor $\mathrm{p}-\mathrm{n}$ junction", Solid-St. Electron, 29, 915-918.

[6] Tang, T.-W. and Gan, H. (2000). "Two formulations of semiconductor transport equations based on spherical harmonic expansion of the Boltzmann transport equations", IEEE Trans. Elec. Dev., 47, 1726-1732.

[7] Bløtekjaer, K. (1970). "Transport equations for electrons in two-valley semiconductors", IEEE Trans. Elec. Dev., ED-17, 38-47.

[8] Tang, T.-W., Ramaswamy, S. and Joonwoo Nam (1993). "An improved hydrodynamic transport model for silicon", IEEE Trans. Elec. Dev., 40, 1469-1477.

[9] Ramaswamy, S. and Tang, T.-W. (1994). "Comparison of semiconductor transport models using a Monte Carlo consistency test", IEEE Trans. Elec. Dev., 41, $76-83$.

[10] Hänsch, W. and Miura-Mattausch, M. (1986). "The hotelectron problem in small semiconductor devices", J. Appl. Phys., 40, 650-656.

[11] Gan, H., "Re-examination of the hot-carrier transport model using spherical harmonic expansion of the Boltzmann transport equation", Master's Thesis, University of Massachusetts, 1999.

[12] Ieong, M. K. and Tang, T.-W. (1997). "Influence of hydrodynamic models in the prediction of submicrometer device characteristics", IEEE Trans. Elec. Dev., 44, $2242-2251$. 

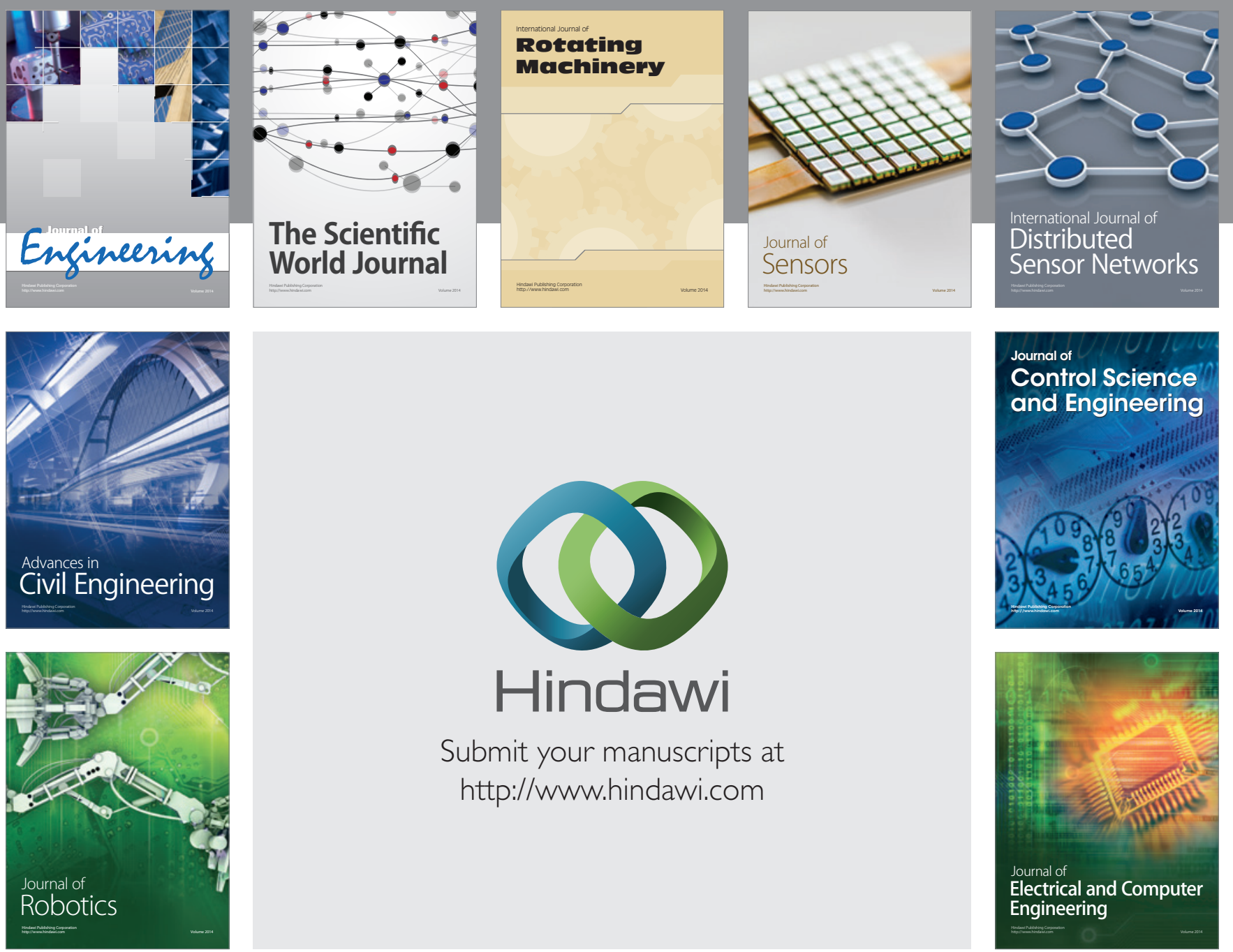

Submit your manuscripts at

http://www.hindawi.com
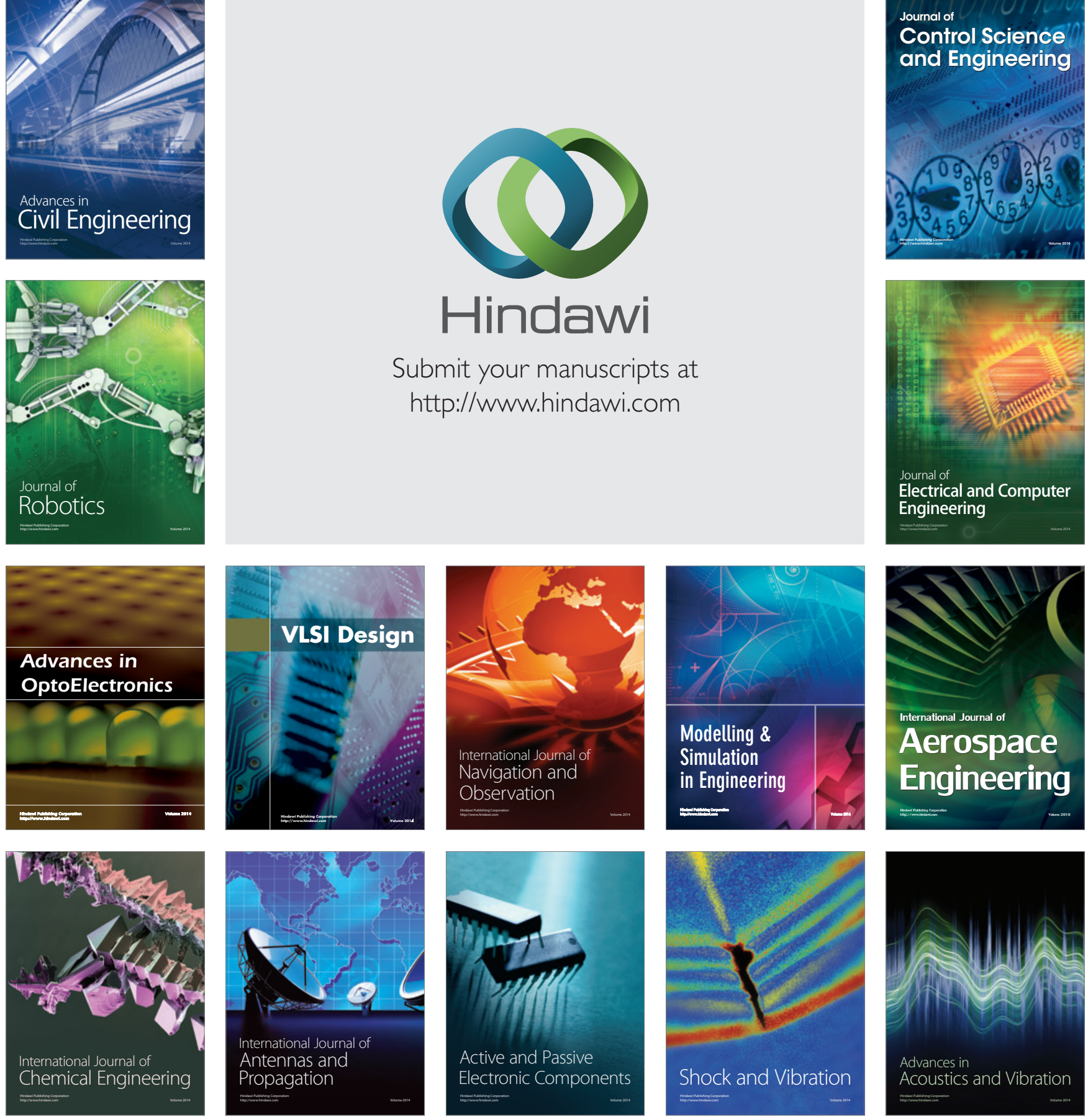